\title{
AN ALGEBRAIC APPROACH TO THE REGULARITY INDEX OF FAT POINTS IN $\mathbf{P}^{n}$
}

\author{
BY NGô VIÊT TRUng
}

\begin{abstract}
The aim of this note is to present an efficient algebraic method for the estimation of the (Castelnuovo) regularity index of fat points in $\mathbf{P}^{n}$ and to discuss recent results on this topic.
\end{abstract}

\section{Introduction}

Given a point $P$ in the projective space $\mathbf{P}^{n}:=\mathbf{P}^{n}(k), k$ an algebraically closed field, we say that a form (or a hypersurface) $f$ of the polynomial ring $R:=k\left[X_{0}, \ldots, X_{n}\right]$ has multiplicity $m$ at $P$ if all derivatives of $f$ of order $\leqq m$ vanish at $P$.

Let $X=\left\{P_{1}, \ldots, P_{s}\right\}$ be a set of $s$ points in $\mathbf{P}^{n}$ and $m_{1} \geqq \ldots \geqq m_{s}$ a sequence of positive integers. We denote by $m_{1} P_{1}+\cdots m_{s} P_{s}$ the zero-scheme defined by the ideal of all forms of $R$ vanishing at $P_{i}$ with multiplicity $\geqq m_{i}, i=1, \ldots, s$, and call

$$
Z:=m_{1} P_{1}+\cdots m_{s} P_{s} .
$$

a set of fat points in $\mathbf{P}^{n}$. There are many reasons for our interest in this notion. For instance, $Z$ is simply the zero-scheme of hypersurfaces passing through $X$ if $m_{1}=\ldots=$ $m_{s}=1$, and of hypersurfaces containing $P_{1}, \ldots, P_{s}$ as singular points if $m_{1}=\ldots=$ $m_{s}=2$.

Let $\mathcal{I}$ denote the ideal sheaf of $Z$. Then $H^{0}\left(\mathbf{P}^{n}, \mathcal{I}(t)\right)$ corresponds to the linear system of hypersurfaces of degree $t$ passing through $P_{1}, \ldots, P_{s}$ with multiplicity $\geqq m_{i}$ at $P_{i}, i=1, \ldots, s$. If $H^{1}\left(\mathbf{P}^{n}, \mathcal{I}(t)\right)=0$, this linear system is called regular. The least integer $t$ for which $H^{1}\left(\mathbf{P}^{n}, \mathcal{I}(t)\right)=0$ is called the regularity index of $Z$, and we will denote it by $r(Z)$. There has been much interest to estimate $r(Z)$ in terms of $m_{1}, \ldots, m_{s}$.

For arbitrary fat points in $\mathbf{P}^{2}$ one can find in W. Fulton [6] the following upper bound:

$$
r(Z) \leqq \sum_{i=1}^{s} m_{i}-1
$$

This bound was extended to arbitrary fat points in $\mathbf{P}^{n}$ by E. Davis and A. Geramita in [4], where they also showed that $r(Z)=\sum_{\imath=1}^{s} m_{i}-1$ if and only if $P_{1}, \ldots, P_{s}$ lie on a line of $\mathbf{P}^{n}$. For almost all sets $X$ of $s$ points in $\mathbf{P}^{2}$, B. Segre [16] found the upper bound:

$$
r(Z) \leqq \max \left\{m_{1}+m_{2}-1,\left[\sum_{\imath=1}^{s} m_{\imath} / 2\right]\right\}
$$

Received May 19, 1993. 
See also G. Paxia [14] for a more rigorous proof. It has turned out that this bound also holds for arbitrary sets of points in general position.

Recall that a set $X$ of points in $\mathbf{P}^{n}$ is said in general positıon if no $n+1$ points of $X$ lie on a hyperplane of $\mathbf{P}^{n}$. It is well-known that the intersections of an $d$-dimensional irreducible projective variety of $\mathbf{P}^{n}$ with a general $(d-n)$-dimensional linear space is a set of points in general position. So in order to study properties of irreducible projective varieties one may study points in general position arising from such intersections.

For fat points in general position in $\mathbf{P}^{2}$, there were first some attemps by $\mathrm{S}$. Greco [9], Davis and Geramita [4] to prove the bound $r(Z) \leqq[s m / 2]$ for points in the case $m_{1}=\ldots=m_{s}=m$. Then M.V. Catalisano [2] succeeded in showing Segre's bound for arbitrary $m_{1}, \ldots, m_{s}$. She also proved that this bound is attained for points lying on a rational normal curve. But her method which has a geometric nature could not be applied to study fat points in $\mathbf{P}^{n}, n$ arbitrary.

Using Castelnuovo's Lemma on the postulation of points in general position in $\mathbf{P}^{n}$ one can easily prove the following upper bound for the regularity index of $X$ :

$$
r(X) \leqq[(s+n-2) / n]
$$

and show that this bound is attained for points lying on a rational normal curve [11] (see [17] and [18] for applications on the defining equations of arithmetically Cohen-Macaulay varieties). This bound suggests that Segre's bound could be generalized to fat points in $\mathbf{P}^{n}, n$ arbitrary.

In this note we will present an algebraic method for the estimation of $r(Z)$ which has helped M.V. Catalisano, G. Valla, and the author [3], [18] to obtain the following generalized version of Segre's bound for arbitrary fat points in general position in $\mathbf{P}^{n}$ :

$$
r(Z) \leqq \max \left\{m_{1}+m_{2}-1,\left[\left(\sum_{i=1}^{s} m_{i}-n+2\right) / n\right]\right\} .
$$

In particular, this bound is attained if $X$ lies on a rational curve, and if $m_{s}$ is large enough, the converse is also true. Moreover, this method can be used to give other good upper bounds for points with uniform position property and generic sets of points.

A set $X$ of points in $\mathbf{P}^{n}$ is said to have the uniform position property (U.P.P. for short) if every two subsets of $X$ with the same cardinality have the same Hilbert function or, equivalently, if the Hilbert function $h_{Y}(t)$ of every subset $Y$ of $X$ verifies the equality

$$
h_{Y}(t)=\min \left\{\# Y, h_{X}(t)\right\}
$$

for all $t \geqq 0$. The significance of this notion comes from Harris' uniform position principle [10] which asserts that if $\operatorname{char}(k)=0$, the points of a general hyperplane section of an irreducible curve are indistinguishable from each other (see [15] for the case $\operatorname{char}(k) \neq 0)$. Since the above bound is also the best upper bound in terms of $m_{1}, \ldots, m_{s}$ for the regularity index of non-degenerate fat points with U.P.P., we will seek upper bounds for $r(Z)$ which involve other numerical invariants of $X$ such as the regularity index $r(X)$ and the Hilbert function $h_{X}(t)$ of $X$.

We say that a property $\mathcal{P}$ holds for a generic set of points in $\mathbf{P}^{n}$ if there exists a nonempty (Zariski) open subset $U$ of the projective space parametrizing the sets of the same number of points in $\mathbf{P}^{n}$ such that $\mathcal{P}$ is satisfied for every set $X \in U$. It is clear that for a generic set of points in $\mathbf{P}^{n}, r(Z)$ has a fixed value depending only on $m_{1}, \ldots, m_{s}$ and the exact value of $r(Z)$ has been known only in the trivial case $m_{1}=\ldots=m_{s}=1$ and 
in the case $m_{1}=\ldots=m_{s}=2$ (see [12] and [11]). This indicates that the computation of $r(Z)$ is very difficult. Nevertheless, using the presented algebraic method we are also able to give an upper bound for the regularity index of generic fat points in $\mathbf{P}^{n}$ which differs from the exact value of $r(Z)$ atmost by 1 in the above case.

We refer the reader to [3] and [18] for the proofs of the results presented here.

\section{The algebraic approach}

Let $\wp_{1}, \ldots, \wp_{s}$ denote the defining prime ideals of $P_{1}, \ldots, P_{s}$ in the polynomial ring $R$, respectively. Put

$$
I:=\wp_{1}^{m_{1}} \cap \ldots \cap \wp^{s} .
$$

Then $A:=R / I$ is the coordinate ring of the zero-scheme $Z$. Since $A$ is an one-dimensional Cohen-Macaulay graded $k$-algebra, the Hilbert function

$$
h_{A}(t):=\operatorname{dim}_{k} A_{t}
$$

is strictly increasing until it reaches the multiplicity $e(A)$ of $A$ at which it stabilizes. Note that $h_{Z}(t)=h_{A}(t)$. The least integer $t$ for which $h_{A}(t)=e(A)$ is called the Castelnuovo regularity index of $A$, and we will denote it by $r(A)$. It is well-known that

$$
r(Z)=r(A) \text {. }
$$

We shall see that one can use induction on $s$ to compute $r(Z)$. Note that the Castelnuovo regularity index can be defined for any graded $k$-algebra [13], [5]. Let

be the defining ideal of the zero-schemes

$$
J:=\wp_{1}^{m_{1}} \cap \ldots \cap \wp_{s-1}^{m_{s-1}}
$$

$$
W:=m_{1} P_{1}+\cdots+m_{s-1} P_{s-1} .
$$

Then $R /\left(J+\wp_{s}^{m_{s}}\right)$ is a zero-dimensional graded algebra and the Castelnuovo regularity index $r\left(R /\left(J+\wp_{s}^{m_{s}}\right)\right)$ is the least integer $t$ for which $\left[R /\left(J+\wp_{s}^{m_{s}}\right)\right]_{t}=0$. The inductive method is based on the following lemma.

LEMMA 1.1. $\quad r(A)=\max \left\{m_{s}-1, r(R / J), r\left(R /\left(J+\wp_{s}^{m_{s}}\right)\right)\right\}$.

This lemma has the following interesting consequence which gives a lower bound for $r(Z)$.

LEMMA $1.2 . \quad r(R / Z) \geq m_{1}+m_{2}-1$.

In Lemma 1.1 one can egive an estimate for $r(R / J)=r(W)$ by the induction hypothesis, where the induction beginningt is guaranteed by the following result.

LEMMA 1.3. Let $X=\left\{P_{1}, \ldots, P_{s}\right\}$ be a set of $s \leqq n+1$ points in general position. Then $r(Z)=m_{1}+m_{2}-1$.

It remains to estimate $r\left(R /\left(J+\wp^{m_{s}}\right)\right)$. For this one can use the following lemma.

LEMMA 1.4. If there exısts a form of degree $t$ in $J$ which does not belong to $\wp_{s}$, then $r\left(R /\left(J+\wp_{s}^{m_{s}}\right)\right) \leqq m_{s}+t-1$. 
So in order to estimate $r(Z)$ one only need to find a hypersurface of an as small as possible degree which passes through the fat points of $W$ but not $P_{s}$. Such a hypersurface can be constructed by considering products of hypersurfaces passing some of the points $P_{1}, \ldots, P_{s}$ but avoiding $P_{s}$. The degree of such a hypersurface will determine the form of the upper bound for $r(Z)$ as we will see in the following sections.

For fat points in $\mathbf{P}^{2}$ there is another efficient algebraic method to bound $r(Z)$ which takes into account the least integer $t$ such that there exists a regular sequence of two forms of degree $t$ in the defining ideal $I(X)$ of $X[19$, Section 3].

\section{Fat points in general position}

Let $X$ be a set of points in general position in $\mathbf{P}^{n}$. To find a hypersurface of an as small as possible degree which passes through the fat points of $W$ but not $P_{s}$ we can take products of hyperplanes passing through $n$ points of $P_{1}, \ldots, P_{s-1}$ but avoiding $P_{s}$. If $t$ is an integer such that $n t \geq \sum_{t=1}^{s-1} m_{i}$ and $t \geq m_{1}$, there are $t$ hyperplanes, say $L_{1}, \ldots, L_{t}$, avoiding $P$ such that $L_{1} \ldots L_{t} \in J$. ¿From this we can deduce the following generalized version of Segre's bound for the regularity index of fat points in $\mathbf{P}^{n}$ :

THEOREM 2.1. Let $P_{1}, \ldots, P_{s}$ be distinct points in general positıon in $\mathbf{P}^{n}$ and $m_{1} \geq \ldots \geq m_{s}$ arbitrary positive integers. Then

$$
r(Z) \leq \max \left\{m_{1}+m_{2}-1,\left[\left(\sum m_{i}+n-2\right) / n\right]\right\} .
$$

We will call this upper bound Segre's bound for the regularity index of fat points in $\mathbf{P}^{n}$. Note that the term $m_{1}+m_{2}-1$ is not important because by Corollary 1.2 it is a lower bound for $r(Z)$.

Using Bezout's Theorem we can prove that Segre's bound is attained for points lying on a rational normal curve.

Proposition 2.2. Let $P_{1}, \ldots, P_{s}$ be distinct points on a rational normal curve in $\mathbf{P}^{n}$ and $m_{1} \geqq \ldots \geqq m_{s}$ arbitrary positive integers. Then

$$
r(Z)=\max \left\{m_{1}+m_{2}-1,\left[\left(\sum m_{i}+n-2\right) / n\right]\right\} \text {. }
$$

This proposition shows that Segre's bound is the best upper bound for $r(Z)$ in terms of $m_{1}, \ldots, m_{s}$.

Segre's bound can be also attained by points not lying on a rational curve [2]. However, if $s \geqq 2 n+3$ and $m_{s}$ is large enough, the converse of Proposition 2.2 is also true, i.e. the given points must lie on a rational normal curve if Segre's bound is attained. Since $m_{1}+m_{2}-1$ is a lower bound for $r(Z)$, the actual question is when $r(Z)=\delta(Z)$.

To prove the converse of Proposition 2.2 we use again the above presented method combined with the classical Castelnuovo's Lemma. This lemma states that for an arbitrary set $X$ of $s$ points in general position in $\mathbf{P}^{n}$,

$$
h_{X}(t) \geqq \min \{s, 2 t+1\}
$$

and if $h_{X}(t)=2 t+1$ for some $t<r(X)$, then $X$ lies on a rational normal curve (see e.g. [11]). Our idea is to involve quadratic forms in order to find a hypersurface of small 
degree which passes through $Y$ but not $P_{s}$ if $X$ does not lie on a rational normal curve. In this case, a quadric could pass more points among $P_{1}, \ldots, P_{s-1}$ than the $2 n$ points of two hyperplanes.

Proposition 2.3. Let $X=\left\{P_{1}, \ldots, P_{s}\right\}, s \geqq 2 n+3$, be a set of points in general position in $\mathbf{P}^{n}$ and $m_{1} \geqq \ldots \geqq m_{s}$ a sequence of positive integers such that

(i) $m_{s} \geqq n$,

(ii) $\delta(Z) \geqq m_{1}+m_{2}+2 n$.

Then $r(Z)=\delta(Z)$ if and only if $X$ lies on a rational normal curve.

The condition $m_{s} \geqq n$ can be lessended by taking into account the residue of $\sum_{\imath=1}^{s} m_{i}-n+2$ modulo $n$ as it has been done in the case $n=2$ by Catalisano [2]. But this will make the statement more complicated.

We conclude this section with two open questions:

Question 1. If $X$ lies on a rational normal curve, does the Hilbert function $h_{Z}(t)$ of the fat points $Z$ depend only on the number of the points and the multiplicities $m_{1}, \ldots, m_{s}$ ? QUESTION 2. If the answer to Question 3.1 is yes, does such a Hilbert function give a lower bound for the Hilbert function of an arbitrary set of fat points in general position with the same number of points and the same multiplicties?

The answers to these questions is yes in the following cases:

a) $m_{1}=\ldots=m_{s}=1, t$ arbitrary (Castelnuovo's Lemma).

b) $m_{1}, \ldots, m_{s}$ arbitrary, $t=r(Z)$ (Theorem 2.1 and Proposition 2.2).

\section{Fat points with uniform position property}

Let $X$ now be a set of points with U.P.P. in $\mathbf{P}^{n}$. Without loss of generality, we may assume that $X$ is non-degenerate, i.e. $X$ lies on no hyperplane of $\mathbf{P}^{n}\left(h_{X}(1)=n+1\right)$. Under this assumption, $X$ is in general position. Hence Segre's bound is also an upper bound for the regularity index of non-degenerate fat points with U.P.P.

On the other hand, if $Y$ is a set of points lying on a rational normal curve, then $h_{Y}(t)=\min \{\# Y, n t+1\}$ for all $t \geqq 0$. Applying this formula to every subset $Y$ of a set $X$ of points on a rational normal curve we obtain $h_{Y}(t)=\min \{\# Y, n t+1\}=$ $\min \left\{\# Y, h_{X}(t)\right.$ hence $X$ has U.P.P.. Therefore, according to Proposition 2.2 Segre's bound is the best upper bound in terms of $m_{1}, \ldots, m_{s}$ for the regularity index of nondegenerate fat points with U.P.P.. For this reason we will seek upper bounds for $r(Z)$ which involve other numerical invariants of $X$ such as the regularity index $d:=r(X)$ and the Hilbert function $h_{i}:=h_{X}(i)$ of $X$.

By the definition of points with U.P.P. there exists, for $i=1, \ldots, d$, a hypersurface of degree $i$ which passes through the first $h_{i}-1$ points of $X$ but avoding $P_{s}$. Considering products of such hypersurfaces we can find a hypersurface of degree $\sum_{b=0}^{d-1} m_{h_{2}}$ which passes through $W$ but not $P_{s}$. Now using the inductive method of Section 1 we can prove the following upper bound for $r(Z)$ :

Theorem 3.1. Let $X=\left\{P_{1}, \ldots, P_{s}\right\}$ be a non-degenerate set of points with U.P.P. in $\mathbf{P}^{n}$ with $h_{\imath}=h_{X}(i), d=r(X)$, and $m_{1} \geqq \ldots \geqq m_{s}$ a sequence of posi- 
tive integers. Then

$$
r(Z) \leqq \max \left\{m_{1}+m_{2}-1, \sum_{i=0}^{d-1} m_{h_{2}}+m_{n+2}-1\right\} .
$$

If $n \geqq 3$ and $m_{1}>1$, then

$$
r(Z) \leqq \max \left\{m_{1}+m_{2}-1, \sum_{i=0}^{d-1} m_{h_{\imath}}+m_{n+3}-1\right\} .
$$

There are examples showing that the bounds of Theorem 2.1 are usually better than Segre's bound.

From Theorem 2.1 we get the following bound for $r(Z)$ which depends only on the regularity index of $X$.

Corollary 3.2. Let $X=\left\{P_{1}, \ldots, P_{s}\right\}$ be a non-degenerate set of points with U.P.P. in $\mathbf{P}^{n}$ with $d=r(X)$ and $m_{1} \geqq \ldots \geqq m_{s}$ a sequence of positive integers. Then

$$
r(Z) \leqq m_{1}+\cdots+m_{d+1}-1 \text {. }
$$

The bound of Corollary 2.2 is sharp for the case $m_{1}=\ldots=m_{s}=1$. Moreover, it could be viewed as a generalisation of the following bound for the regularity index of generic fat points in $\mathbf{P}^{2}$ given by Gimigliano [8, Theorem 1]:

$$
r(Z) \leqq m_{1}+\cdots+m_{d} .
$$

Note that Gimigliano's bound does not hold for generic fat points in $\mathbf{P}^{n}$ with $n \geqq 3$.

For generic fat points, $r(Z)$ has a fixed value which depends only on $m_{1}, \ldots, m_{s}$. It is clear that the Hilbert function of a generic set $X$ of $s$ points in $\mathbf{P}^{n}$ is as large as possible, i.e.

$$
h_{X}(i)=\min \left\{s,\left(\begin{array}{c}
n+i \\
n
\end{array}\right)\right\}
$$

and hence

$$
r(X)=\min \left\{t: s \leqq\left(\begin{array}{c}
n+t \\
n
\end{array}\right)\right\} .
$$

Except this trivial case $Z=X\left(m_{1}=\ldots=m_{s}=1\right)$, to compute the concrete value of $r(Z)$ seems to be very difficult. It is known only in the non-trivial case $m_{1}=\ldots=m_{s}=2$ by Hirschowitz [12] and Alexander [1]. Using the inductive method of Section 1 we can also prove the following bound for the regularity index of generic fat points (with arbitrary $\left.m_{1}, \ldots, m_{s}\right)$ which differs from the exact value of $r(Z)$ at most by 1 in the case $m_{1}=\ldots=m_{s}=2$.

Theorem 3.3. Let $X=\left\{P_{1}, \ldots, P_{s}\right\}$ be a generic set of points in $\mathbf{P}^{n}$ and $m_{1} \geqq$ $\ldots \geqq m_{s}$ be a sequence of positive integers. Let $w$ denote the least integer such that

$$
\sum_{i=2}^{s}\left(\begin{array}{c}
m_{i}+n-1 \\
n
\end{array}\right)<\left(\begin{array}{c}
n+w \\
n
\end{array}\right)
$$




$$
r(Z) \leqq m_{1}+w-1
$$

The proof relied on the facts that one may find a hypersurface of degree $w$ which passes through the zero-scheme $m_{2} P_{2}+\cdots+m_{s} P_{s}$ but not $P_{1}$.

For generic sets of points, the bound of Theorem 2.3 is usually better than the bound of Theorem 2.1 and Gimiliano's bound. In particular, if $m_{1}=\ldots=m_{s}=2$, we have by Theorem $2.3 r(Z) \leqq w+1$, where $w$ is the least integer for which $(s-1)(n+1)<\left(\begin{array}{c}n+w \\ n\end{array}\right)$. Since

$$
\left(\begin{array}{c}
n+w-1 \\
n
\end{array}\right)=h_{R}(w-1) \leqq(s-1)(n+1)<s(n+1)=e(R / I),
$$

$w \leqq r(Z) \leqq w+1$. Hence the bound $w+1$ differs from $r(Z)$ at most by 1 .

\section{REFERENCE}

[1 ] J. Alexander, Singularités imposable en position general à une hypersurface projective, Compos. Math. 68 (1988), 305-354.

[2] M.V. Catalisano, Linear systems of plane curves through fixed "fat" points in $\mathbf{P}^{2}$, J. Algebra 142 (1991), 81-100.

[ 3 ] M.V. Catalisano, N.V. Trung, and G. Valla, A sharp bound for the regularity index of fat points in general position, Proc. Amer. Math. Soc., to appear.

[4] E.D. Davis and A.V. Geramita, The Hilbert function of a special class of 1-dimensional CohenMacaulay graded algebras, The Curves Seminar at Queen's, Queen's Papers in Pure and Appl. Math. 67 (1984),1-29.

[ 5 ] D. Eisenbud and S. Goto, Linear free resolutions and minimal multiplicity, J. Algebra 88 (1984), 89-133.

[6] W. Fulton, Algebraic Curves, Math. Lect. Note Series, Benjamin 1969.

[7] A. Gimigliano, Our thin knowledge on fat points, in: The Curve's Seminar at Queen's VI, Queen's Papers in Pure and Appl. Math. 83 (1989).

[ 8 ] A.Gimigliano, Regularity of linear systems of plane curves, J. Algebra 124 (1989), 447-460.

[ 9 ] S. Greco, Remarks on the postulation of zero-dimensional subschemes of projective spaces, Math. Ann 284 (1989), 343-351.

[10] J. Harris, A bound on the geometric genus of projective varieties, Ann. Sc. Norm. Sup. Pisa Ser. IV. 8 (1981), 35-68.

[11] J. Harris (with the collaboration of D. Eisenbud), Curves in projective spaces, Les Presses de l'Universite de Montreal 1982.

[12] A. Hirschowitz, La methode d'Horace pour l'Interpolation à plusieurs variables, Manus. Math. 50 (1985), 337-388.

[13] A. Ooishi, Castelnuovo's regularity of graded rings and modules, Hiroshima Math. J. 12 (1982), 627-649.

[14] G. Paxia, On flat families of fat points, Proc. Amer. Math. Soc. 112 (1991), 19-23.

[15] J. Rathmann, The uniform position principle for curves in charcteristic $p$, Math. Ann. 276 (1987), 565-579.

[16] B. Segre, Alcune questioni su insiemi finiti di punti in geometria algebrica, Atti. Convergno. Intern. di Torino 1961, 15-33.

[17] R. Treger, On equations defining arithmetically Cohen-Macaulay schemes I, Math. Ann. 261 (1982), 141-153. 
[18] N.V. Trung and G. Valla, Degree bounds for the defining equations of arithmetically CohenMacaulay curves, Math. Ann. 281 (1988), 209-218.

[19] N.V. Trung and G. Valla, Upper bounds for the regularity index of fat points with uniform position property, Preprint.

INSTITUTE OF MATHEMATICS

Box 631, Bò Hô, Hanoi, Vietnam 